\title{
On the Kesava Menon norm of semimultiplicative functions
}

\author{
Pentti HaukKanen
}

\begin{abstract}
The Kesava Menon norm of an arithmetical function $f$ is defined by $N(f)(n)=$ $(f * \lambda f)\left(n^{2}\right)$, where $*$ denotes the Dirichlet convolution and $\lambda$ denotes Liouville's function. The $m$ th power Kesava Menon norm of $f$ is defined inductively by $N^{0}(f)=f, N^{m}(f)=$ $N\left(N^{m-1}(f)\right), m=1,2, \ldots$ In this paper we prove that the $m$ th power Kesava Menon norm of a semimultiplicative function is semimultiplicative and that the $m$ th power Kesava Menon norm distributes over the Dirichlet convolution of semimultiplicative functions. In addition we show that the $m$ th power Kesava Menon norm of a rational arithmetical function of degree $(r, s)$ is a rational arithmetical function of the same degree.
\end{abstract}

Mathematics Subject Classification. 11A25.

Keywords. Semimultiplicative function, Kesava Menon norm, Dirichlet convolution, Rational arithmetical function.

\section{Introduction}

Let $f$ be an arithmetical function (that is, a real- or complex-valued function on the set of positive integers). In 1963, Kesava Menon [4, Section 3] defined the norm of $f$ as the arithmetical function $N(f)$ given by

$$
N(f)(n)=(f * \bar{f})\left(n^{2}\right),
$$

where $*$ is the Dirichlet convolution (see $(1))$ and $\bar{f}$ is the conjugate of $f$. The conjugate is defined as

$$
\bar{f}=\lambda f,
$$

where $\lambda$ is Liouville's function (see (3)). The norm $N(f)$ is referred to as the Kesava Menon norm in the literature [6, Section 5]. Redmond and Sivaramakrishnan [9, Section 4] defined the $m$ th power Kesava Menon norm of $f$ inductively by 


$$
\begin{aligned}
N^{0}(f) & =f \\
N^{m}(f) & =N\left(N^{m-1}(f)\right) \quad \text { for } m=1,2, \ldots
\end{aligned}
$$

In this paper we investigate the conjugate, the Kesava Menon norm and the $m$ th power Kesava Menon norm of semimultiplicative functions. Semimultiplicative functions form a superclass of the class of the usual multiplicative functions. Quasimultiplicative functions lie between multiplicative and semimultiplicative functions. Rational arithmetical functions form the subgroup of the group of multiplicative functions under the Dirichlet convolution generated by completely multiplicative functions. An arithmetical function $f$ is said to be a rational arithmetical function of degree $(r, s)$ if it is the Dirichlet convolution of $r$ completely multiplicative functions and the inverse of $s$ completely multiplicative functions. For details of these various types of multiplicativity, see Sect. 2.

This paper is organized as follows. In Sect. 2 we review the known properties of arithmetical functions needed in this paper. In Sect. 3 we present new results. In Sect. 3 we first note that the conjugate of a semimultiplicative function is semimultiplicative and that the conjugate distributes over the Dirichlet convolution of any two arithmetical functions. We continue by applying these results to show that the $m$ th power Kesava Menon norm of a semimultiplicative function is semimultiplicative, that is, the $m$ th power Kesava Menon norm preserves semimultiplicativity. As special cases we obtain the same properties for quasimultiplicative and multiplicative functions. Therefore our result generalizes the result of Sivaramakrishnan [11, Section 2], namely that the usual Kesava Menon norm of a multiplicative function is multiplicative. In Sect. 3 we also prove that the $m$ th power Kesava Menon norm distributes over the Dirichlet convolution of semimultiplicative functions, extending the result of Laohakosol and Pabhapote [6, Section 5], who proved that the $m$ th power Kesava Menon norm distributes over the Dirichlet convolution of rational arithmetical functions. We apply our distributivity property to show that the $m$ th power Kesava Menon norm preserves the Dirichlet inverse of a quasimultiplicative function.

In Sect. 4 of this paper we utilize the properties presented in Sect. 3 to prove that the $m$ th power Kesava Menon norm of a rational arithmetical function of degree $(r, s)$ is a rational arithmetical function of the same degree. Laohakosol and Pabhapote [6, Section 5] proved the same result in a different way. We also present the analogous results for the conjugate of semimultiplicative functions and rational arithmetical functions of degree $(r, s)$. 


\section{Preliminaries on arithmetical functions}

The Dirichlet convolution of arithmetical functions $f$ and $g$ is defined as

$$
(f * g)(n)=\sum_{d \mid n} f(d) g(n / d) .
$$

We may also interpret that an arithmetical function $f$ is defined on the set of positive real numbers so that $f(x)=0$ if $x$ is not a positive integer. This makes it possible to present the Dirichlet convolution in the form

$$
(f * g)(n)=\sum_{k=1}^{\infty} f(k) g(n / k) .
$$

This expression is useful in some calculations presented in this paper. The function $\delta$, defined as $\delta(1)=1$ and $\delta(n)=0$ otherwise, serves as the identity under the Dirichlet convolution. The Dirichlet inverse of $f$ exists if and only if $f(1) \neq 0$, and it is denoted by $f^{-1}$.

An arithmetical function $f$ is said to be multiplicative if $f(1)=1$ and $f(m n)=f(m) f(n)$ for all coprime positive integers $m, n$. An arithmetical function $f$ is said to be semimultiplicative [8] if there exists a nonzero constant $c_{f}$, a positive integer $a_{f}$ and a multiplicative function $f_{M}$ such that

$$
f(n)=c_{f} f_{M}\left(n / a_{f}\right) .
$$

Semimultiplicative functions can also be characterized as the arithmetical functions $f$ (not identically zero) satisfying the functional equation

$$
f(m) f(n)=f((m, n)) f([m, n])
$$

for all positive integers $m$ and $n$, where $(m, n)$ and $[m, n]$ are the gcd and lcm of $m$ and $n$. Semimultiplicative functions are the same as Selberg multiplicative functions (see [2, Section 2.1] and [10]). We do not present the details here.

Quasimultiplicative functions (see [2, Section 2.1] and [12, Section XI.2]) are the arithmetical functions $f$ such that $f(1) \neq 0$ and $f(1) f(m n)=f(m) f(n)$ for all coprime positive integers $m, n$. Lahiri [5] refers to these functions as hypo-multiplicative functions. Quasimultiplicative functions are, in fact, the semimultiplicative functions $f$ with $a_{f}=1$ (i.e. with $\left.f(1) \neq 0\right), c_{f}=f(1)$ and $f_{M}(n)=f(n) / f(1)$. Note that $f$ is multiplicative if and only if $f$ is semimultiplicative with $a_{f}=c_{f}=1$ and $f_{M}=f$.

The Dirichlet convolution of multiplicative functions is multiplicative. The same applies to quasimultiplicative and semimultiplicative functions. To be more precise [8, Section 5], if $f$ and $g$ are semimultiplicative, then $f * g$ is semimultiplicative with

$$
c_{f * g}=c_{f} c_{g}, \quad a_{f * g}=a_{f} a_{g}, \quad(f * g)_{M}=f_{M} * g_{M} .
$$


A multiplicative function $f$ is said to be completely multiplicative if $f(m n)=f(m) f(n)$ for all positive integers $m, n$. Liouville's function $\lambda$ is an example of a completely multiplicative function. It is defined as

$$
\lambda(n)=(-1)^{\Omega(n)},
$$

where $\Omega(n)$ represents the total number of prime factors of $n$, each counted according to multiplicity [1, Section 2.12].

A multiplicative function $f$ is said to be a rational arithmetical function of degree $(r, s)$ if

$$
f=g_{1} * \cdots * g_{r} *\left(h_{1} * \cdots * h_{s}\right)^{-1}
$$

for some completely multiplicative functions $g_{1}, \ldots, g_{r}, h_{1}, \ldots, h_{s}$ (see [6] and [13, Section III]). A rational arithmetical function of degree $(2,0)$ is referred to as a specially multiplicative function [9]. If $f=g_{1} * g_{2}$ is a specially multiplicative function, we denote $f_{A}=g_{1} g_{2}$. The function $f_{A}$ is termed as the associated completely multiplicative function. For example, the divisor functions $\sigma_{a}$ and Ramanujan's $\tau$-function are specially multiplicative functions. Euler's totient function $\phi$ is a rational arithmetical function of degree $(1,1)$.

For general accounts on arithmetical functions, we refer to $[1,7,12]$.

\section{The $m$ th power Kesava Menon norm of semimultiplicative functions}

In this section we first note in Theorems 3.1 and 3.2 that the conjugate of a semimultiplicative function is semimultiplicative and that the conjugate distributes over the Dirichlet convolution of any two arithmetical functions. We then apply these theorems to prove Theorems 3.3, 3.4 and 3.5, which state that the $m$ th power Kesava Menon norm of a semimultiplicative function is semimultiplicative and that the $m$ th power Kesava Menon norm distributes over the Dirichlet convolution of semimultiplicative functions.

Theorem 3.1. If $f$ is semimultiplicative, then $\bar{f}$ is semimultiplicative with $c_{\bar{f}}=$ $\lambda\left(a_{f}\right) c_{f}, a_{\bar{f}}=a_{f}$ and $(\bar{f})_{M}=\overline{\left(f_{M}\right)}=\lambda f_{M}$.

Theorem 3.2. For all arithmetical functions $f$ and $g$,

$$
\overline{f * g}=\bar{f} * \bar{g} .
$$

Theorems 3.1 and 3.2 follow directly from the definitions of conjugate and semimultiplicative function and from complete multiplicativity of $\lambda$.

In order to prove that the $m$ th power Kesava Menon norm preserves semimultiplicativity, we first present this result in the case $m=1$, since this case is needed in various stages of the proof of the general case. 
Theorem 3.3. If $f$ is semimultiplicative, then $N(f)$ is semimultiplicative with

$$
\begin{aligned}
c_{N(f)} & =c_{f} c_{\bar{f}}=\lambda\left(a_{f}\right)\left(c_{f}\right)^{2} \\
a_{N(f)} & =a_{f} \\
(N(f))_{M} & =N\left(f_{M}\right) .
\end{aligned}
$$

Proof. By the definitions of the Kesava Menon norm and the Dirichlet convolution,

$$
N(f)(n)=(f * \bar{f})\left(n^{2}\right)=\sum_{k=1}^{\infty} f(k) \bar{f}\left(n^{2} / k\right) .
$$

Applying the definition of a semimultiplicative function and Theorem 3.1, we obtain

$$
\begin{aligned}
N(f)(n) & =\sum_{k=1}^{\infty} c_{f} f_{M}\left(k / a_{f}\right) \lambda\left(a_{f}\right) c_{f}\left(\lambda f_{M}\right)\left(n^{2} /\left(k a_{f}\right)\right) \\
& =\lambda\left(a_{f}\right)\left(c_{f}\right)^{2} \sum_{k=1}^{\infty} f_{M}(k)\left(\lambda f_{M}\right)\left(\left(n^{2} /\left(a_{f}\right)^{2}\right) / k\right) \\
& =\lambda\left(a_{f}\right)\left(c_{f}\right)^{2}\left(f_{M} *\left(\lambda f_{M}\right)\right)\left(\left(n / a_{f}\right)^{2}\right) .
\end{aligned}
$$

By the definitions of the conjugate and the Kesava Menon norm, we see that

$$
N(f)(n)=\lambda\left(a_{f}\right)\left(c_{f}\right)^{2} N\left(f_{M}\right)\left(n / a_{f}\right) .
$$

By Theorem 3.1, $\lambda\left(a_{f}\right)\left(c_{f}\right)^{2}=c_{f} c_{\bar{f}}(\neq 0)$. Since the Kesava Menon norm of a multiplicative function is multiplicative, $N\left(f_{M}\right)$ is multiplicative. We thus obtain the result.

Theorem 3.4. If $f$ is semimultiplicative, then $N^{m}(f)$ (where $m \geq 0$ ) is semimultiplicative with

$$
\begin{aligned}
c_{N^{m}}(f) & =c_{f}\left(c_{\bar{f}}\right)^{2^{m}-1}=\left(\lambda\left(a_{f}\right)\right)^{2^{m}-1}\left(c_{f}\right)^{2^{m}} \\
a_{N^{m}}(f) & =a_{f} \\
\left(N^{m}(f)\right)_{M} & =N^{m}\left(f_{M}\right) .
\end{aligned}
$$

Proof. We proceed by induction on $m$. For $m=0$ the theorem holds, since $N^{0}(f)=f$. The case $m=1$ is presented in Theorem 3.3.

Suppose that the theorem is true for $m=k$. Thus $N^{k}(f)$ is semimultiplicative, and then applying Theorem 3.3 we see that $N\left(N^{k}(f)\right)$ is semimultiplicative, that is, the function $N^{k+1}(f)$ is semimultiplicative.

Further, from Theorem 3.3, we have

$$
c_{N^{k+1}(f)}=c_{N\left(N^{k}(f)\right)}=\lambda\left(a_{N^{k}(f)}\right)\left(c_{N^{k}(f)}\right)^{2} .
$$

By the induction hypothesis,

$$
c_{N^{k+1}(f)}=\lambda\left(a_{f}\right)\left(\left(\lambda\left(a_{f}\right)\right)^{2^{k}-1}\left(c_{f}\right)^{2^{k}}\right)^{2}=\left(\lambda\left(a_{f}\right)\right)^{2^{k+1}-1}\left(c_{f}\right)^{2^{k+1}} .
$$


By Theorem 3.3 and the induction hypothesis,

$$
a_{N^{k+1}(f)}=a_{N\left(N^{k}(f)\right)}=a_{N^{k}(f)}=a_{f}
$$

and

$$
\begin{aligned}
\left(N^{k+1}(f)\right)_{M} & =\left(N\left(N^{k}(f)\right)\right)_{M}=N\left(\left(N^{k}(f)\right)_{M}\right) \\
& =N\left(N^{k}\left(f_{M}\right)\right)=N^{k+1}\left(f_{M}\right) .
\end{aligned}
$$

This completes the proof.

Corollary 3.1. If $f$ is quasimultiplicative, then $N^{m}(f)$ (where $m \geq 0$ ) is quasimultiplicative with $N^{m}(f)(1)=f(1)^{2^{m}}$.

Proof. If $f$ is quasimultiplicative, then $a_{f}=1$, and thus

$$
a_{N^{m}(f)}=a_{f}=1 \text {. }
$$

This shows that $N^{m}(f)$ is quasimultiplicative. Since $\lambda\left(a_{f}\right)=\lambda(1)=1$,

$$
N^{m}(f)(1)=c_{N^{m}(f)}=\left(\lambda\left(a_{f}\right)\right)^{2^{m}-1}\left(c_{f}\right)^{2^{m}}=\left(c_{f}\right)^{2^{m}}=f(1)^{2^{m}} .
$$

This completes the proof.

Corollary 3.2. If $f$ is multiplicative, then $N^{m}(f)$ (where $m \geq 0$ ) is multiplicative.

Corollary 3.2 follows directly from Corollary 3.1, since each multiplicative function $f$ is quasimultiplicative with $f(1)=1$.

Theorem 3.5. If $f$ and $g$ are semimultiplicative, then

$$
N^{m}(f * g)=N^{m}(f) * N^{m}(g), \quad m \geq 0 .
$$

Proof. Suppose first that $f$ and $g$ are multiplicative. Then, by the definition of the Kesava Menon norm, for all prime powers $p^{e}$,

$$
N(f * g)\left(p^{e}\right)=((f * g) * \overline{(f * g)})\left(p^{2 e}\right) .
$$

By Theorem 3.2, we obtain

$$
N(f * g)\left(p^{e}\right)=((f * \bar{f}) *(g * \bar{g}))\left(p^{2 e}\right)=\sum_{i=0}^{2 e}(f * \bar{f})\left(p^{i}\right)(g * \bar{g})\left(p^{2 e-i}\right) .
$$

But $(f * \bar{f})\left(p^{i}\right)=0$ if $i$ is odd; hence we have

$$
\begin{aligned}
N(f * g)\left(p^{e}\right) & =\sum_{i=0}^{e}(f * \bar{f})\left(p^{2 i}\right)(g * \bar{g})\left(p^{2(e-i)}\right) \\
& =\sum_{i=0}^{e} N(f)\left(p^{i}\right) N(g)\left(p^{e-i}\right)=(N(f) * N(g))\left(p^{e}\right) .
\end{aligned}
$$


Since $f$ and $g$ are multiplicative, $N(f * g)$ and $N(f) * N(g)$ are also multiplicative. A multiplicative function is totally determined by its values at prime powers. Therefore

$$
N(f * g)=N(f) * N(g) .
$$

Now, applying induction on $m$ gives

$$
N^{m}(f * g)=N^{m}(f) * N^{m}(g) .
$$

Consider now the general case that $f$ and $g$ are semimultiplicative. Then, by Theorem 3.4 and Eq. (2), $N^{m}(f * g)$ and $N^{m}(f) * N^{m}(g)$ are semimultiplicative. In addition, using Theorem 3.4 we have

$$
c_{N^{m}(f * g)}=\left(\lambda\left(a_{f * g}\right)\right)^{2^{m}-1}\left(c_{f * g}\right)^{2^{m}} .
$$

On the basis of (2),

$$
c_{N^{m}(f * g)}=\left(\lambda\left(a_{f} a_{g}\right)\right)^{2^{m}-1}\left(c_{f} c_{g}\right)^{2^{m}} .
$$

Since $\lambda$ is completely multiplicative, $\lambda\left(a_{f} a_{g}\right)=\lambda\left(a_{f}\right) \lambda\left(a_{g}\right)$. Therefore

$$
c_{N^{m}}(f * g)=\left(\lambda\left(a_{f}\right)\right)^{2^{m}-1}\left(c_{f}\right)^{2^{m}}\left(\lambda\left(a_{g}\right)\right)^{2^{m}-1}\left(c_{g}\right)^{2^{m}} .
$$

Using Theorem 3.4 and Eq. (2) we get

$$
c_{N^{m}(f * g)}=c_{N^{m}(f)} c_{N^{m}(g)}=c_{\left(N^{m}(f) * N^{m}(g)\right)} .
$$

Further, applying Theorem 3.4 and Eq. (2) we get

$$
\begin{aligned}
a_{N^{m}(f * g)} & =a_{f * g}=a_{f} a_{g} \\
& =a_{N^{m}(f)} a_{N^{m}(g)} \\
& =a_{\left(N^{m}(f) * N^{m}(g)\right)} .
\end{aligned}
$$

Next, applying Theorem 3.4 and Eq. (2), we get

$$
\left(N^{m}(f * g)\right)_{M}=N^{m}\left((f * g)_{M}\right)=N^{m}\left(f_{M} * g_{M}\right) .
$$

On the basis of the first part of this proof on multiplicative functions,

$$
\left(N^{m}(f * g)\right)_{M}=\left(N^{m}\left(f_{M}\right)\right) *\left(N^{m}\left(g_{M}\right)\right) .
$$

By Theorem 3.4 and Eq. (2),

$$
\left(N^{m}(f * g)\right)_{M}=\left(N^{m}(f)\right)_{M} *\left(N^{m}(g)\right)_{M}=\left(N^{m}(f) * N^{m}(g)\right)_{M} .
$$

Finally, combining (4), (5) and (6) gives

$$
N^{m}(f * g)=N^{m}(f) * N^{m}(g) .
$$

This completes the proof.

Corollary 3.3. If $f$ and $g$ are quasimultiplicative, then

$$
N^{m}(f * g)=N^{m}(f) * N^{m}(g), \quad m \geq 0 .
$$


Corollary 3.4. If $f$ and $g$ are multiplicative, then

$$
N^{m}(f * g)=N^{m}(f) * N^{m}(g), \quad m \geq 0 .
$$

Corollaries 3.3 and 3.4 follow directly from Theorem 3.5, since each quasimultiplicative function is semimultiplicative and each multiplicative function is quasimultiplicative.

Theorem 3.6. If $f$ is quasimultiplicative, then

$$
N^{m}\left(f^{-1}\right)=\left(N^{m}(f)\right)^{-1}, \quad m \geq 0 .
$$

Proof. By Theorem 3.5,

$$
N^{m}(f) * N^{m}\left(f^{-1}\right)=N^{m}\left(f * f^{-1}\right)=N^{m}(\delta) .
$$

Now,

$$
N(\delta)=\delta * \bar{\delta}=\delta * \delta=\delta .
$$

Applying induction, we obtain

$$
N^{m}(\delta)=\delta
$$

This completes the proof.

Corollary 3.5. If $f$ is multiplicative, then

$$
N^{m}\left(f^{-1}\right)=\left(N^{m}(f)\right)^{-1} .
$$

Corollary 3.5 follows directly from Theorem 3.6, since each multiplicative function is quasimultiplicative.

Theorem 3.7. For all arithmetical functions with $f(1) \neq 0$,

$$
\overline{\left(f^{-1}\right)}=(\bar{f})^{-1} \text {. }
$$

Proof. We have

$$
\bar{f} * \overline{\left(f^{-1}\right)}=\lambda f * \lambda f^{-1}=\lambda\left(f * f^{-1}\right)=\lambda \delta=\delta .
$$

\section{The $m$ th power Kesava Menon norm of rational arithmetical functions}

Laohakosol and Pabhapote [6] proved that the $m$ th power Kesava Menon norm of a rational arithmetical function of degree $(r, s)$ is also a rational arithmetical function of degree $(r, s)$. In this paper we present a short proof (applying Corollaries 3.4 and 3.5; see the proof of Theorem 4.1). Redmond and Sivaramakrishnan [9] proved this result for rational arithmetical functions of degree $(2,0)$, that is, for specially multiplicative functions. We note in Corollary 4.2 a similar result for the conjugate of a rational arithmetical function of degree $(r, s)$. 
Theorem 4.1. Suppose that $f$ is a rational arithmetical function of degree $(r, s)$ given as

$$
f=g_{1} * \cdots * g_{r} *\left(h_{1} * \cdots * h_{s}\right)^{-1},
$$

where $g_{1}, \ldots, g_{r}, h_{1}, \ldots, h_{s}$ are completely multiplicative functions. Then $N^{m}(f)$ is a rational arithmetical function of degree $(r, s)$ such that

$$
N^{m}(f)=\left(g_{1}\right)^{2^{m}} * \cdots *\left(g_{r}\right)^{2^{m}} *\left(\left(h_{1}\right)^{2^{m}} * \cdots *\left(h_{s}\right)^{2^{m}}\right)^{-1}, \quad m \geq 0 .
$$

Proof. For a completely multiplicative function $g$ we have

$$
N(g)\left(p^{e}\right)=(g *(\lambda g))\left(p^{2 e}\right)=(g(u * \lambda))\left(p^{2 e}\right)
$$

for all prime powers $p^{e}$, where $u(n)=1$ for all positive integers $n$. Here

$$
(u * \lambda)\left(p^{2 e}\right)=\sum_{i=0}^{2 e}(-1)^{2 e-i}=\sum_{i=0}^{2 e}(-1)^{i}=1 .
$$

Therefore

$$
N(g)\left(p^{e}\right)=g\left(p^{2 e}\right)=g^{2}\left(p^{e}\right) .
$$

Since $N(g)$ and $g^{2}$ are multiplicative functions, this implies

$$
N(g)=g^{2} .
$$

Applying induction on $m$ gives

$$
N^{m}(g)=g^{2^{m}}
$$

Now, by Corollaries 3.4 and 3.5, we obtain Theorem 4.1.

Corollary 4.1. Suppose that $f$ is a specially multiplicative function. Then $N^{m}(f)$ is specially multiplicative with $\left(N^{m}(f)\right)_{A}=\left(f_{A}\right)^{2^{m}}$. Further,

$$
\left(N^{m}(f)\right)_{A}=N^{m}\left(f_{A}\right) .
$$

Proof. Let $f=g_{1} * g_{2}$, where $g_{1}$ and $g_{2}$ are completely multiplicative functions. Then $f_{A}=g_{1} g_{2}$. On the other hand, by Theorem 4.1, $N^{m}(f)=g_{1}^{2^{m}} * g_{2}^{2^{m}}$, and thus

$$
\left(N^{m}(f)\right)_{A}=g_{1}^{2^{m}} g_{2}^{2^{m}}=\left(g_{1} g_{2}\right)^{2^{m}}=\left(f_{A}\right)^{2^{m}} .
$$

Further, since $f_{A}$ is completely multiplicative, by Theorem 4.1, we obtain

$$
N^{m}\left(f_{A}\right)=\left(f_{A}\right)^{2^{m}} .
$$

This completes the proof. 
Example 4.1. The divisor function $\sigma_{a}$ and Jordan's totient function $J_{a}$ are defined by

$$
\begin{aligned}
\sigma_{a} & =\operatorname{Id}_{a} * u \\
J_{a} & =\operatorname{Id}_{a} * u^{-1}=\operatorname{Id}_{a} * \mu,
\end{aligned}
$$

where $\operatorname{Id}_{a}(n)=n^{a}$ is the power function and $\mu$ is the Möbius function. Thus we have

$$
\begin{aligned}
& N^{m}\left(\sigma_{a}\right)=\left(\operatorname{Id}_{a}\right)^{2^{m}} * u^{2^{m}}=\operatorname{Id}_{2^{m} a} * u=\sigma_{2^{m} a} \\
& N^{m}\left(J_{a}\right)=\left(\operatorname{Id}_{a}\right)^{2^{m}} *\left(u^{2^{m}}\right)^{-1}=\operatorname{Id}_{2^{m}} * u^{-1}=\operatorname{Id}_{2^{m}} a \mu=J_{2^{m}} a .
\end{aligned}
$$

In particular, $J_{1}$ is the well-known Euler's totient function $\phi$, and thus

$$
N^{m}(\phi)=J_{2^{m}}
$$

Theorem 4.2. Let $f$ be an arithmetical function given as

$$
f=g_{1} * \cdots * g_{r} * h_{1}^{-1} * \cdots * h_{s}^{-1},
$$

where $h_{1}, h_{2}, \ldots, h_{s}$ are arithmetical functions such that $h_{1}(1), \ldots, h_{s}(1) \neq 0$. Then the conjugate of $f$ is given as

$$
\bar{f}=\overline{g_{1}} * \cdots * \overline{g_{r}} *\left(\overline{h_{1}}\right)^{-1} * \cdots *\left(\overline{h_{s}}\right)^{-1} .
$$

Proof. The conjugate of $f$ is

$$
\bar{f}=\lambda\left(g_{1} * \cdots * g_{r} *\left(h_{1} * \cdots * h_{s}\right)^{-1}\right)=\lambda\left(g_{1} * \cdots * g_{r} * h_{1}^{-1} * \cdots * h_{s}^{-1}\right) .
$$

The usual product of a completely multiplicative function distributes over the Dirichlet product. Thus, since $\lambda$ is completely multiplicative,

$$
\bar{f}=\left(\lambda g_{1}\right) * \cdots *\left(\lambda g_{r}\right) *\left(\lambda h_{1}^{-1}\right) * \cdots *\left(\lambda h_{s}^{-1}\right) .
$$

By the definition of the conjugate,

$$
\bar{f}=\overline{g_{1}} * \cdots * \overline{g_{r}} * \overline{h_{1}^{-1}} * \cdots * \overline{h_{s}^{-1}} .
$$

Now, applying Theorem 3.7, we obtain Theorem 4.2.

Corollary 4.2. If $f$ is a rational arithmetical function of degree $(r, s)$, then its conjugate is also a rational arithmetical function of degree $(r, s)$ (given as in Theorem 4.2).

Proof. The conjugate of a completely multiplicative function is completely multiplicative. Therefore this corollary follows from Theorem 4.2.

\section{Acknowledgements}

The author wishes to thank the referee for useful comments. 
Open Access. This article is distributed under the terms of the Creative Commons Attribution 4.0 International License (http://creativecommons.org/licenses/by/4.0/), which permits unrestricted use, distribution, and reproduction in any medium, provided you give appropriate credit to the original author(s) and the source, provide a link to the Creative Commons license, and indicate if changes were made.

Publisher's Note Springer Nature remains neutral with regard to jurisdictional claims in published maps and institutional affiliations.

\section{References}

[1] Apostol, T.M.: Introduction to Analytic Number Theory. Springer, Berlin (1976)

[2] Haukkanen, P.: Extensions of the class of multiplicative functions. East-West J. Math. 14(2), 101-113 (2012)

[3] Haukkanen, P.: Embedding the unitary divisor meet semilattice in a lattice. Notes Number Theory Discrete Math. 22(3), 68-78 (2016)

[4] Kesava Menon, P.: Series associated with Ramanujans's function $\tau(n)$. J. Indian Math. Soc. 27, 57-65 (1963)

[5] Lahiri, D.B.: Hypo-multiplicative number-theoretic functions. Aequationes Math. 9, 184-192 (1973)

[6] Laohakosol, V., Pabhapote, N.: Properties of rational arithmetic functions. Int. J. Math. Math. Sci. 2005(24), 3997-4017 (2005)

[7] McCarthy, P.J.: Introduction to Arithmetical Functions, Universitext. Springer, Berlin (1986)

[8] Rearick, D.: Semi-multiplicative functions. Duke Math. J. 33, 49-53 (1966)

[9] Redmond, D., Sivaramakrishnan, R.: Some properties of specially multiplicative functions. J. Number Theory 13, 210-227 (1981)

[10] Selberg, A.: Remarks on multiplicative functions. In: Proceedings of the Conference on Number Theory Day at Rockefeller University New York, 1976, pp. 232-241. Springer, (1977)

[11] Sivaramakrishnan, R.: On a class of multiplicative arithmetic functions. J. Reine Angew. Math 280, 157-162 (1976)

[12] Sivaramakrishnan, R.: Classical Theory of Arithmetic Functions, Monographs and Textbooks in Pure and Applied Mathematics, vol. 126. Marcel Dekker Inc., New York (1986)

[13] Vaidyanathaswamy, R.: The theory of multiplicative arithmetical functions. Trans. Amer. Math. Soc. 33, 579-662 (1931)

Pentti Haukkanen

Faculty of Information Technology and Communication Sciences

Tampere University

FI-33014 Tampere

Finland

e-mail: pentti.haukkane@tuni.fi

Received: January 22, 2019

Revised: June 14, 2019 